\title{
riccafd
}

Revista Iberoamericana de Ciencias de la Actividad Fisica y el Deporte

\section{BENEFICIOS DEL ENTRENAMIENTO VIBRATORIO EN EL SISTEMA NEUROMUSCULAR DE LOS ADULTOS MAYORES}

\section{BENEFITS OF VIBRATION TRAINING IN THE NEUROMUSCULAR SYSTEM OF THE ELDERLY}

\section{Palop-Montoro, $\mathrm{MV}^{1}$; Arteaga-Checa, $\mathrm{M}^{2}$; Lozano-Aguilera, $\mathrm{E}^{3}$ y Párraga- Montilla, JA ${ }^{4}$}

1 Profesora doctora en Fisioterapia, Universidad de Jaén, España, mpalop@ujaen.es

${ }^{2}$ Profesora Titular de Universidad, doctora en Ciencias de la Actividad Física y del Deporte, Universidad de Jaén, España, marteaga@ujaen.es

3 Profesor Titular de Universidad, doctor en Estadística e Investigación Operativa, Universidad de Jaén, España, elozano@ujaen.es

${ }^{4}$ Profesor Titular de Universidad, doctor en Ciencias de la Actividad Física y del Deporte, Universidad de Jaén, España, jparraga@ujaen.es

Código UNESCO: 3201.07 Geriatría; 3207.99 Otras patologías (Sarcopenia) Clasificación Consejo de Europa: 17. Otras (Educación Física y Salud)

Recibido el 13 de junio de 2015

Aceptado el 28 de octubre de 2015

Correspondencia:

Palop-Montoro, MV: mpalop@ujaen.es

\section{RESUMEN}

Introducción: El entrenamiento vibratorio puede ser una opción de intervención óptima para prevenir la pérdida de masa muscular en adultos mayores.

Objetivo: Comprobar la efectividad del entrenamiento vibratorio en el sistema neuromuscular de los adultos mayores.

Material y método: Revisión bibliográfica en las bases de datos Medline, CINAHL, WOS y PEDro, mediante la combinación de los descriptores del Medical Subjects Headings referentes a entrenamiento vibratorio, fuerza muscular, masa muscular y personas mayores.

Resultados: Los estudios localizados sobre el entrenamiento vibratorio en personas mayores se realizaron, bien como única intervención, o en combinación con otro tipo de ejercicios de fuerza, aeróbicos, de equilibrio o de 
flexibilidad. La diversidad de protocolos empleados dificulta una comparación entre estudios.

Conclusión: El entrenamiento con plataformas vibratorias demuestra ser un método de entrenamiento de la fuerza seguro, adecuado y eficaz para la población de mayor edad, pero con resultados similares al ejercicio de resistencia convencional.

PALABRAS CLAVE: Vibraciones de cuerpo completo, fuerza muscular, masa muscular, adultos mayores.

\section{ABSTRACT}

Introduction: The vibration training may be an option for optimal intervention to prevent muscle loss in older adults.

Objective: To assess the effectiveness of vibration training in the neuromuscular system of the elderly.

Methods: Literature review in Medline, CINAHL, WOS and PEDro data by combining the descriptors of Medical Subject Headings concerning vibration training, muscle strength, muscle mass and seniors.

Results: Studies located on the vibration training in older people were done as either the only intervention, or in combination with other strength exercises, aerobic, balance or flexibility. The diversity of protocols hinders comparison between studies.

Conclusion: WBV training proves to be a healthy method, adequate and effective in the strength of the elderly population, but with similar results to conventional resistance exercise.

KEYWORDS: Whole body vibration, muscle strength, muscle mass, older people.

\section{INTRODUCCIÓN}

El envejecimiento humano está asociado a una pérdida de masa muscular que se atribuye al descenso de fibras musculares ${ }^{1}$. Surge como un proceso natural y universal que contribuye a una disminución de la capacidad funcional e independencia en las actividades de la vida diaria y afecta a la calidad de vida de las personas mayores. Algunos estudios señalan que si bien la pérdida de masa muscular relativa es más temprana, situándose a la edad de 30 años, la masa muscular absoluta no comienza a descender hasta la quinta década de vida; siendo mayor en las extremidades inferiores que en las superiores ${ }^{2}$ y en varones más que en mujeres ${ }^{3}$.

La pérdida de masa muscular y, por tanto de fuerza, afecta al $20 \%$ de los varones entre 70 y 75 años, al $50 \%$ de los de más de 80 años y entre el 25 y el $40 \%$ de las mujeres, en las mismas franjas de edad ${ }^{4}$. Entre sus causas podemos destacar las alteraciones en la síntesis y degradación de proteínas, la 
inflamación, las alteraciones hormonales y la disfunción mitocondrial ${ }^{5-9}$. La actividad física puede determinar su evolución, de forma que la inactividad produce una mayor y más rápida pérdida de masa muscular. Así, existe evidencia de la eficacia del ejercicio físico para disminuir la pérdida de masa magra y mejora de la fuerza muscular, siendo la principal opción como intervención no farmacológica. Un ejercicio alternativo, las vibraciones de cuerpo completo o whole body vibration (WBV), surgió como una potencial intervención que podría influir positivamente en el sistema muscular de los adultos mayores.

Las vibraciones estimulan el sistema neuromuscular, activando la musculatura a través de los reflejos espinales. Como consecuencia de la detección de vibraciones por parte de los husos neuromusculares se provoca un aumento de la ratio de descarga de estas estructuras ${ }^{10}$. Esto produce un aumento de los potenciales motores evocados en los músculos sometidos a vibración ${ }^{11-12}$, lo que supone una activación de los circuitos medulares, en los que se basa el reflejo miotático ${ }^{13}$. Pero el efecto de la estimulación vibratoria no se limita a estructuras medulares. El aumento de los potenciales motores y de la frecuencia de señal electromiográfica, tras una exposición prolongada, sugieren un estado de notable excitabilidad de la corteza motora y provocaría un mayor reclutamiento de fibras tipo $\mathrm{II}^{14}$. Por tanto, puede suponer un estado de mayor eficiencia neuromuscular que permite mayor rendimiento en los movimientos voluntarios ${ }^{15-16}$.

Se ha de tener muy en cuenta la frecuencia, amplitud, dirección y duración de las vibraciones, ya que el cuerpo humano ha demostrado responder de manera altamente específica a la variación de estos parámetros ${ }^{17}$. Actualmente se especula que cada colectivo puede requerir de unos parámetros específicos diferentes, al igual que cada individuo debe responder de un modo a la aplicación de vibraciones $\mathrm{y}$, por tanto, deberían prescribirse de forma personalizada. Es más, cada grupo muscular parece tener una frecuencia de estimulación específica.

La eficacia de WBV reside en su facilidad de uso y el poco tiempo requerido de utilización. Puede servir para la mejora de la eficiencia neuromuscular y, sus efectos positivos ya observados en adultos jóvenes, conllevan la necesidad de un mayor conocimiento de la posible utilización de WBV en una población de más edad. Las investigaciones sugieren que WBV puede afectar positivamente el rendimiento muscular ${ }^{18-19}$. Otros estudios, sin embargo, han reportado resultados equívocos ${ }^{20-21}$. La diversidad de los protocolos de entrenamiento vibratorio empleados hace que la comparación de las intervenciones sea extremadamente difícil.

\section{OBJETIVO}

El propósito de este estudio es investigar los efectos de WBV sobre el sistema neuromuscular de los adultos mayores. 


\section{METODOLOGÍA}

El diseño de este estudio es de tipo descriptivo transversal de artículos publicados mediante una revisión bibliográfica. La fuente de obtención de datos se obtuvo de la consulta directa a la literatura científica indizada en las siguientes bases de datos: Medical Literature Analysis and Retrieval System Online (MEDLINE), Cumulative Index to Nursing and Allied Health Literature (CINAHL), Web Of Science (WOS) y Physiotherapy Evidence Database (PEDro).

Se analizaron los artículos publicados cuyo idioma fuera inglés, portugués o castellano; acotando la búsqueda desde el año 2000 en adelante. Los descriptores utilizados fueron: "whole body vibration", "vibration training", "older people", "elderly persons", "muscle strength", "muscle mass" y "lean mass"; mediante su combinación con los operadores booleanos AND y OR. Los límites empleados fueron: humanos y ensayos clínicos. Los estudios debían estar publicados como originales en revistas documentadas, fueron seleccionados a partir del título y resumen de los mismos, y se obtuvieron a texto completo para un análisis más detallado. La fecha de la última actualización de la búsqueda se realizó en diciembre de 2014. Los documentos debieron cumplir con los siguientes criterios:

- Criterios de inclusión: Ensayos clínicos aleatorizados publicados en revistas indizadas en bases de datos internacionales, sujetas a revisión por pares y con acceso al texto completo.

- Criterios de exclusión: Revisiones y documentos de interés que no basaban su estudio en personas mayores, que el entrenamiento realizado no fuera específicamente con vibraciones de cuerpo completo, que analizasen el efecto agudo tras una sola sesión y aquellos que no incluyeran como variable de estudio la fuerza o la masa muscular.

\section{RESULTADOS}

Los artículos localizados sobre el entrenamiento vibratorio en personas mayores fueron realizados, bien como única intervención o en combinación con otros ejercicios de fuerza, aeróbicos, de equilibrio o flexibilidad; de los cuales presentamos el resumen de aquellos que cumplían con los criterios de selección.

Runge et al $^{22}$ encontrando un aumento significativo promedio de un $18 \%$ en el test de levantarse de la silla (5-Chair Stands), indicador de la potencia del tren inferior, después de 2 meses de entrenamiento ( 3 días por semana; 3 series de 2 minutos), en un grupo mixto de personas mayores (edad media: 67 años). Russo et al $^{23}$ investigaron el efecto de 6 meses de entrenamiento, por medio de WBV, en 14 mujeres posmenopáusicas con respecto a 15 mujeres de similares características incluidas en un grupo control. El grupo de entrenamiento realizó 2 sesiones semanales que incluían 3 series de 2 minutos de trabajo con 1 
minuto de recuperación ( $28 \mathrm{~Hz}$ de frecuencia; amplitud variable). Después de la intervención, el grupo que realizó el entrenamiento mejoró un $5 \%$ su potencia respecto al grupo control, que incluso lo disminuyó con respecto a sus niveles iniciales; sin embargo, la fuerza muscular no se vio afectada.

Bogaerts et $\mathrm{al}^{24}$ apreciaron mejoras en la fuerza muscular realizando un entrenamiento con WBV durante un año. El protocolo consistió en $30-40 \mathrm{~Hz}$ de frecuencia, 2,5-5 mm de amplitud y 1-3 series por ejercicio; ejecutando 8 posiciones estáticas diferentes. La masa muscular de los participantes también se vio beneficiada. Del mismo modo, Roelants et $a^{19}$, tras 6 meses de entrenamiento vibratorio, 3 veces por semana, reportaron mejoras en la fuerza muscular y velocidad de movimiento de los extensores de la rodilla, así como en la capacidad de salto de mujeres posmenopáusicas (58-74 años de edad). No obstante, similares mejoras fueron reportadas por el grupo que realizó un entrenamiento de fuerza convencional, y en ambos grupos se produjeron fundamentalmente en las primeras 12 semanas. Los parámetros empleados en el estudio fueron: $35-40 \mathrm{~Hz}$ de frecuencia; $2,5-5 \mathrm{~mm}$ de amplitud; y 1-3 series de 30-60 s de ejercicio con 60-5 s de reposo. Los ejercicios fueron enfocados hacia los extensores de piernas y variaron de 2 a 9 durante el transcurso de la intervención.

Iwamoto et $\mathrm{al}^{25}$ evaluaron la eficacia de un programa de WBV en la deambulación de 25 mujeres mayores. El entrenamiento incluía una sesión semanal de 4 minutos a $20 \mathrm{~Hz}$ de frecuencia y $0,7-4,2 \mathrm{~mm}$ de amplitud; complementado con ejercicios diarios de equilibrio estático y de fuerza, consistentes en "flamingos" y 10 sentadillas (sin peso adicional). Después de 3 meses, se observaron mejoras significativas en las pruebas de longitud del paso, máximo momento extensor de la rodilla y máximo tiempo mantenido sobre una pierna. Por su parte, Furness et $\mathrm{al}^{26}$ dividieron a una muestra de 73 adultos mayores en 4 grupos con $0,1,2$ y 3 sesiones de vibración a la semana, encontrando un aumento del rendimiento neuromuscular medido con las pruebas 5-Chair Stands, Timed Up and Go y el test de Tinetti; aunque la mejora más beneficiosa la obtuvo el grupo de 3 sesiones semanales.

Kawanabe et $\mathrm{al}^{27}$ midieron la fuerza muscular y la habilidad para caminar tras 2 meses de WBV (12-20 Hz de frecuencia, 4 minutos de estimulación, 1 vez/semana), con participantes de edad media 72,0 años. No se apreciaron efectos solo con la vibración, pero sí al complementarse con el ejercicio de caminar; matizando en sus resultados que fue bien tolerada por las personas mayores. Del mismo modo, Rees et $a^{28}$ también limitaron su estudio a 2 meses, 3 veces/semana, encontrando mejoras en la musculatura y la movilidad de los sujetos; no obstante, el grupo que realizó ejercicios de fuerza sin vibración tuvo similares resultados. Los mismos investigadores, al año siguiente, realizaron modificaciones en su protocolo, el cual consistió en $26 \mathrm{~Hz}$ de frecuencia y 5-8 $\mathrm{mm}$ de amplitud; parámetros que fueron progresivamente incrementándose, al igual que las 6 series de estímulo consistentes en $45-80 \mathrm{~s}$ de ejercicio y 45-80 s de reposo. La única diferencia entre los grupos experimentales (WBV y entrenamiento de fuerza clásico) la obtuvieron en la fuerza de flexión plantar, que fue mayor en el grupo con entrenamiento 
vibratorio. Raimundo et $\mathrm{al}^{29}$ concluyeron sus investigaciones resaltando que sería beneficioso complementar el entrenamiento vibratorio con el ejercicio de caminar. Trabajaron con mujeres posmenopáusicas (edad media 66,2 años) y sus hallazgos mostraron que WBV previene la disminución de la fuerza en miembros inferiores. La intervención tuvo como parámetros: $12,6 \mathrm{~Hz}$ de frecuencia, $3 \mathrm{~mm}$ de amplitud, 3 veces/semana y 6 series 1:1; durante 8 meses.

Un ensayo clínico se planteó como objetivo examinar los efectos de la postura en bipedestación durante el entrenamiento vibratorio sobre la función muscular y su morfología. Los 19 mayores que formaron la muestra fueron divididos en 3 grupos; unos adoptaron la postura en flexión de rodillas, otros en extensión y el grupo control se mantuvo en flexión sin vibraciones. Después de 3 meses ( 3 veces/semana), la velocidad de contracción de la parte superior del cuerpo mejoró significativamente después de WBV con flexión de rodillas y la fuerza del tren inferior mejoró con ambas posturas, respecto al grupo control. Así, la hipótesis de la adaptación diferencial de la postura solo se observó para la velocidad de miembros superiores ${ }^{30}$.

Recientemente en nuestro país, el grupo de investigación GENUD quiso comprobar si un programa de entrenamiento vibratorio de corta duración tiene algún efecto sobre la masa magra, medida con DXA, en personas mayores. Tras 11 semanas de WBV, los autores señalaron que este periodo no es suficiente para producir cambios significativos. La muestra estuvo formada por 49 mayores no institucionalizados (20 hombres y 29 mujeres) los cuales fueron divididos en dos grupos. 24 personas se sometieron a vibraciones verticales, 3 veces por semana, con una frecuencia de $40 \mathrm{~Hz}$ y una amplitud de $2 \mathrm{~mm}$. Estos sujetos realizaron 10 repeticiones de 45 segundos en posición de squat y 60 segundos de descanso entre cada repetición. Frente a este grupo, el control no realizó ningún entrenamiento. Los autores comunicaron que no hubo efectos adversos ni problemas de salud en los participantes de ambos grupos $^{31}$.

Kennis et $\mathrm{al}^{32}$ aportaron que WBV puede ser un entrenamiento específico para las fibras rápidas. Estos autores evaluaron los efectos de un año de entrenamiento vibratorio en el volumen y calidad muscular, fuerza isométrica y concéntrica, y en la potencia de adultos mayores. Un grupo realizó ejercicios aeróbicos (caminar, correr o cicloergómetro) y de fuerza, y otro grupo entrenamiento vibratorio progresivo, con ejercicios estáticos y dinámicos de piernas sobre la plataforma; ambos 3 veces por semana. Lo interesante de este estudio es su seguimiento después de la intervención, ya que un año después, el grupo de WBV disminuyó la potencia, pero se mantuvo significativamente más alta que en sus valores de inicio del entrenamiento, respecto al grupo de ejercicio y a un control.

Merriman et $\mathrm{al}^{33}$ quisieron precisar la influencia de la edad, el sexo, los hertzios y la duración del efecto en varios indicadores de la función física en adultos mayores residentes en la comunidad. Los participantes fueron expuestos a 2 
minutos de vibración por sesión, con $2 \mathrm{~Hz}$ y $26 \mathrm{~Hz}$. Se registraron las medidas de resultado a los 2, 20 y 40 minutos después de la vibración. Los test "Timed Up-and-Go" (TUG) y "Chair-Sit and Reach" mejoraron tras WBV para ambos sexos, pero fueron significativamente diferentes para los sujetos del estudio entre las frecuencias señaladas. No fue estadísticamente significativa la relación entre TUG y la edad; sí, en cambio, entre TUG y el género. El rendimiento mejoró en hombres más siguiente al tratamiento de $26 \mathrm{~Hz}$, mientras que el rendimiento de las mujeres mejoró más después del tratamiento con $2 \mathrm{~Hz}$. Además, hubo una tendencia general hacia los hombres en mejoras en comparación con las mujeres, independientemente de la condición de tratamiento.

En la tabla 1 presentamos un resumen de los estudios incluidos en esta revisión. 


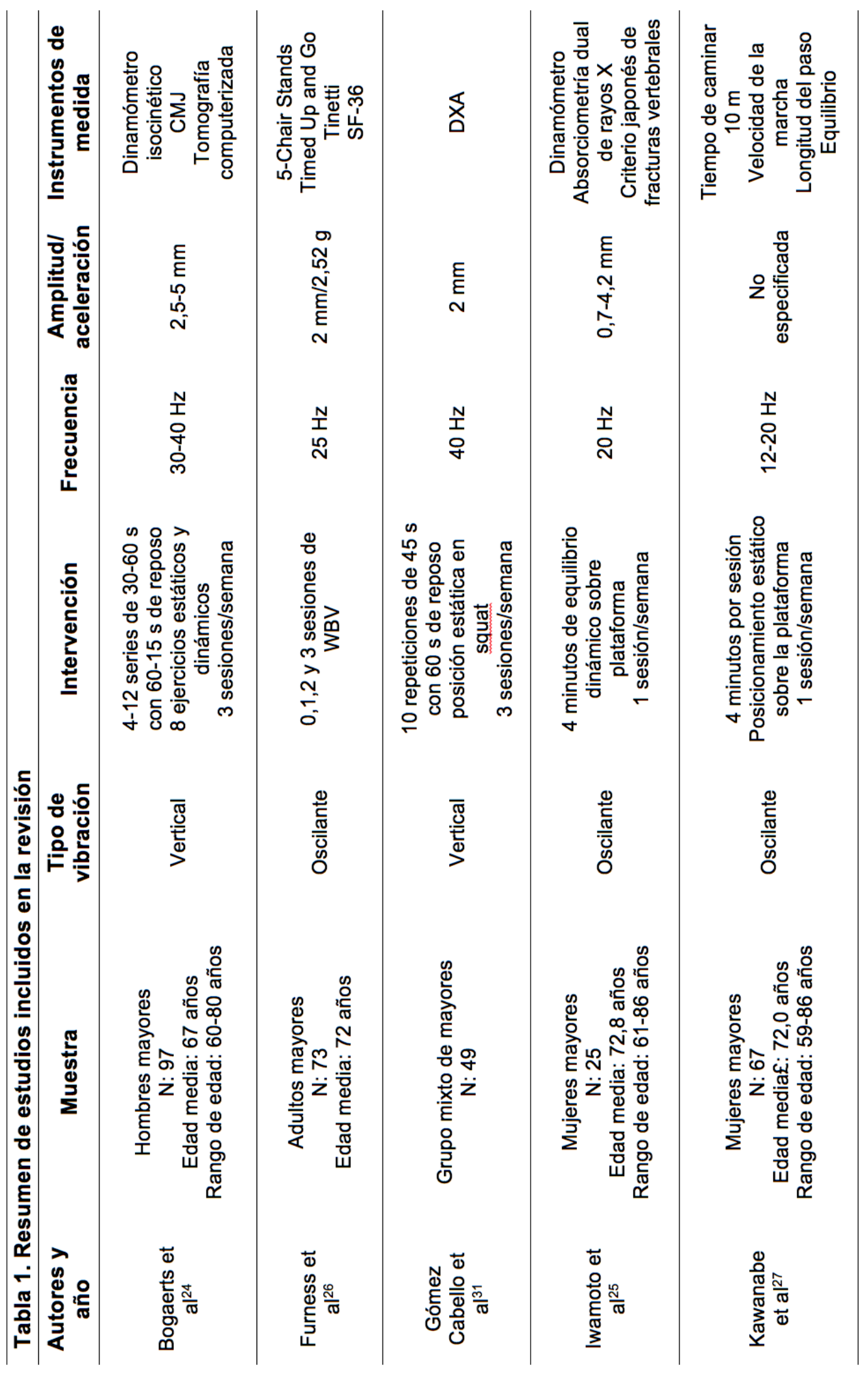




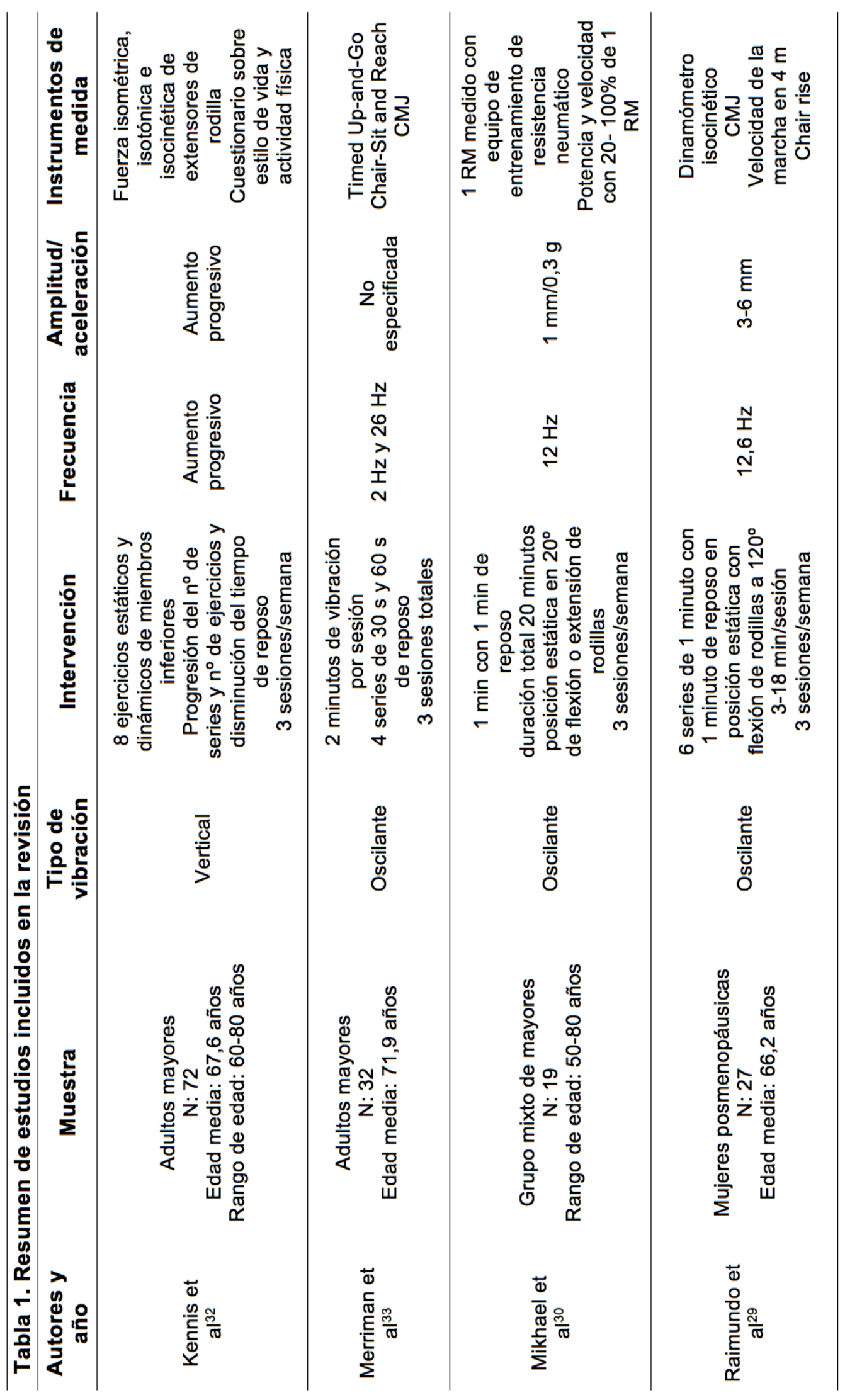




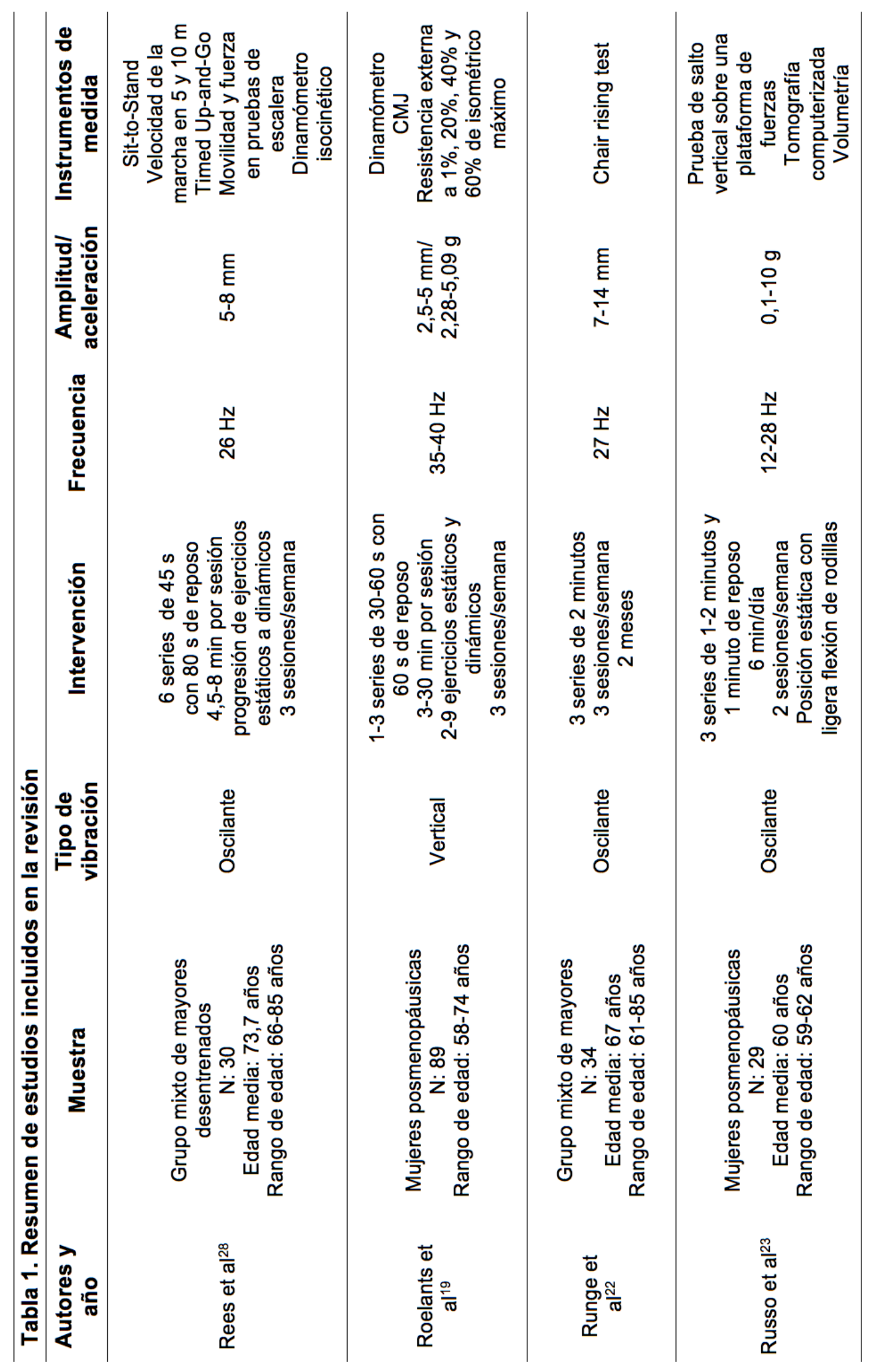




\section{DISCUSIÓN}

Este estudio ofrece una visión de la eficacia de WBV como intervención de capacitación para el desempeño funcional, en adultos mayores, a través de mejoras en el sistema neuromuscular.

Los diferentes resultados que se han analizado podrían ser por la variedad en cuanto a protocolos empleados, la duración de los mismos y las características de la muestra. Así, los estudios que encontraron hipertrofia significativa escogieron a personas mayores institucionalizadas, las cuales estarían más debilitadas. Diversos estudios indican que las mejoras se producen especialmente en aquellos más frágiles y en desentrenados ${ }^{31,34}$. Sitjà-Rabert et $\mathrm{al}^{35}$ supusieron que WBV debe mejorar la fuerza y la potencia muscular de este colectivo cuando se compara con un grupo control; en cambio, los resultados no difieren de los que produce el ejercicio convencional.

Previamente otros investigadores habían testado el impacto de WBV por sí solo (es decir, no combinado con otros tipos de ejercicio) en la masa muscular. Ambos obtuvieron similares incrementos de dicha masa muscular, unos trabajando con mujeres mayores durante 10 semanas $^{36}$, y otros con hombres mayores durante un $a \tilde{n}^{24}$. Los autores manifestaron que en la mayoría de estudios con WBV y adultos mayores predomina el sexo femenino en la muestra o este se realiza exclusivamente con féminas. Este sesgo de género puede ser debido al creciente estudio con mujeres posmenopáusicas así como el mayor porcentaje de mujeres que llegan a edades avanzadas. Solo unos pocos estudios han incluido en su mayoría a hombres mayores ${ }^{37,24,26}$. Ya que los hombres y mujeres de cualquier edad pueden responder de forma diferente a WBV, esta apreciación se ha mantenido en gran parte inexplorada. 


\section{CONCLUSIONES}

WBV demuestra ser un método de entrenamiento de la fuerza seguro, adecuado y eficaz para la población de mayor edad, pero con resultados similares al ejercicio de resistencia convencional. Debido a que este entrenamiento puede llevarse a cabo en el hogar, podría servir como una intervención de ejercicio alternativo en aquellos adultos mayores que no se sienten atraídos por el ejercicio de resistencia tradicional o que no pueden realizarlo debido a alguna afección que los limita de su práctica. Tras la revisión de ensayos clínicos, lo que todos señalan es que este tipo de ejercicio podría ser efectivo en el tratamiento y la prevención de la pérdida muscular asociada a la edad. A pesar de la variabilidad de protocolos empleados en los diferentes estudios, lo que todos refieren en común es que WBV es un método que no produce efectos adversos ni problemas de salud en las personas de edad.

\section{REFERENCIAS}

1. Lexell J, Taylor CC, Sjöström M. What is the cause of the ageing atrophy? Total number, size and proportion of different fiber types studied in whole vastus lateralis muscle from 15 - to 83 -year-old men. J Neurol Sci. 1988;84(2-3):275-94.

2. Janssen I, Heymsfield SB, Wang ZM, Ross R. Skeletal muscle mass and distribution in 468 men and women aged 18-88 yr. J Appl Physiol. 2000;89(1):81-8.

3. Visser M, Kritchevsky SB, Goodpaster BH, Newman AB, Nevitt M, Stamm $\mathrm{E}$ et al. Leg muscle mass and composition in relation to lower extremity performance in men and women aged 70 to 79 : the health, aging and body composition study. J Am Geriatr Soc. 2002;50:897-904.

4. Baumgartner RN, Koehler KM, Gallagher D, Romero L, Heymsfield SB, Ross RR et al. Epidemiology of sarcopenia among the elderly in New Mexico. Am J Epidemiol. 1998;147:755-63.

5. Balagopal P, Schimke JC, Ades P, Adey D, Nair KS. Age effect on transcript levels and synthesis rate of muscle MHC and response to resistance exercise. Am J Physiol Endocrinol Metab. 2001;280(2):E2038.

6. Castillo EM, Goodman-Gruen D, Kritz-Silverstein D, Morton DJ, Wingard $\mathrm{DL}$, Barrett-Connor E. Sarcopenia in elderly men and women: the Rancho Bernardo study. Am J Prev Med. 2003;25:226-31. 
7. Janssen YM, Van Houten B, Borm PJ, Mossman BT. Cell and tissue responses to oxidative damage. Lab Invest. 1993;69(3):261-74.

8. Ji LL, Gómez-Cabrera MC, Vina J. Role of free radicals and antioxidant signaling in skeletal muscle health and pathology. Infect Disord Drug Targets. 2009;9:428-44.

9. Melton LJ, Khosla S, Crowson CS, O'Connor MK, O'Fallon WM, Riggs BL. Epidemiology of sarcopenia. J Am Geriatr Soc. 2000;48:625-30.

10. Nishihira Y, Iwasaki T, Hatta A, Wasaka T, Kaneda T, Kuroiwa K et al. Effect of whole body vibration stimulus and voluntary contraction on motoneuron pool. Adv Exerc Sports Physiol. 2002;8:83-6.

11. Kossev A, Siggelkow S, Schubert M, Wohlfarth K, Dengler R. Muscle vibration: different effects on transcranial magnetic and electrical stimulation. Muscle Nerve. 1999;22:946-8.

12. Kossev A, Siggelkow S, Kapels H, Dengler R, Rollnik JD. Crossed effects of muscle vibration on motor-evoked potentials. Clin Neurophysiol. 2001;112:453-6.

13. Rittweger J, Mutschelknauss $M$, Felsenberg D. Acute changes in neuromuscular excitability after exhaustive whole body vibration exercise as compared to exhaustion by squatting exercise. Clin Physiol Funct Imaging. 2003;23:81-6.

14. Romaiguere $P$, Vedel JP, Pagni S. Effects of tonic vibration reflex on motor unit recruitment in human wrist extensor muscles. Brain Res. 1993;602(1):32-40.

15. Bosco $C$, Colli $R$, Introini E, Cardinale $M$, Tsarpela $O$, Madella $A$ et al. Adaptive responses of human skeletal muscle to vibration exposure. Clin Physiol. 1999;19:183-7.

16. Bosco $\mathrm{C}$, lacovelli $\mathrm{M}$, Tsarpela $\mathrm{O}$, Cardinale $\mathrm{M}$, Bonifazi $\mathrm{M}$, Tihanyi $\mathrm{J}$ et al. Hormonal responses to whole-body vibration in men. Eur $\mathrm{J}$ Appl Physiol. 2000;81:449-54.

17. Cardinale $\mathrm{M}$, Pope $\mathrm{MH}$. The effects of whole body vibration on humans: dangerous or advantageous? Act Physiol Hung. 2003;90:195-206.

18. Delecluse $C$, Roelants $M$, Verschueren S. Strength increase after wholebody vibration compared with resistance training. Med Sci Sports Exerc. 2003;35:1033-41.

19. Roelants M, Delecluse C, Verschueren SM. Whole-body-vibration training increases knee-extension strength and speed of movement in older women. J Am Geriatr Soc. 2004;52:901-8.

20. De Ruiter CJ, Raak SM, Schilperoot JV, Hollander AP, Haan A. The effects of 11 weeks whole body vibration training on jump height, contractile properties and activation of human knee extensors. Eur J Appl Physiol. 2003;88:472-5.

21. Schlumberger A, Salin D, Schmidtbleicher D. Strength training with superimposed vibrations. Sportverletz Sportschaden. 2001;15(1):1-7.

22. Runge M, Rehfeld G, Resnicek E. Balance training and exercise in geriatric patients. J Musculoskel Neuron Interact. 2000;1(1):61-5.

23. Russo CR, Lauretani F, Bandinelli S, Bartali B, Cavazzini C, Guralnik JM et al. High-frequency vibration training increases muscle power in postmenopausal women. Arch Phys Med Rehabil. 2003;84:1854-7. 
24. Bogaerts A, Verschueren S, Delecluse C, Claessens AL, Boonen S. Effects of whole body vibration training on postural control in older individuals: a 1 year randomized controlled trial. Gait Posture. 2007;26:309-16. http://dx.doi.org/10.1016/j.gaitpost.2006.09.078

25. Iwamoto J, Otaka Y, Kudo K, Takeda T, Uzawa M, Hirabayashi K. Efficacy of training program for ambulatory competence in elderly women. Keio J Med. 2004;53:85-9.

26. Furness TP, Maschette WE. Influence of whole body vibration platform frequency on neuromuscular performance of community-dwelling older adults. J Strength Cond Res. 2009;23(5):1508-13. http://dx.doi.org/ 10.1519/JSC.0b013e3181a4e8f9

27. Kawanabe K, Kawashima A, Sashimoto I, Takeda T, Sato Y, Iwamoto J. Effect of whole-body vibration exercise and muscle strengthening, balance, and walking exercises on walking ability in the elderly. Keio $\mathrm{J}$ Med. 2007;56(1):28-33.

28. Rees SS, Murphy AJ, Watsford ML. Effects of whole-body vibration exercise on lower-extremity muscle strength and power in an older population: a randomized clinical trial. Phys Ther. 2008;88(4):462-70. http://dx.doi.org/10.2522/ptj.20070027

29. Raimundo AM, Gusi N, Tomas-Carus P. Fitness efficacy of vibratory exercise compared to walking in postmenopausal women. Eur J Appl Physiol. 2009;106(5):741-8. http://dx.doi.org/10.1007/s00421-009-1067-9

30. Mikhael M, Orr R, Amsen F, Greene D, Singh MA. Effect of standing posture during whole body vibration training on muscle morphology and function in older adults: a randomised controlled trial. BMC Geriatr. 2010; 10:74. http://dx.doi.org/10.1186/1471-2318-10-74

31. Gómez-Cabello A, González-Agüero A, Ara I, Casajus JA, VicenteRodríguez G. Effects of a short-term whole body vibration intervention on physical fitness in elderly people. Maturitas. 2013;74(3):276-8.

32. Kennis E, Verschueren SM, Bogaerts A, Van Roie E, Boonen S, Delecluse C. Long-term impact of strength training on muscle strength characteristics in older adults. Arch Phys Med Rehabil. 2013;94(11):2054-60. http://dx.doi.org/10.1016/j.apmr.2013.06.018

33. Merriman HL, Brahler CJ, Jackson K. Systematically controlling for the influence of age, sex, hertz and time post-whole-body vibration exposure on four measures of physical performance in community-dwelling older adults: a randomized cross-over study. Curr Gerontol Geriatr Res. 2011;2011:747094. http://dx.doi.org/10.1155/2011/747094

34. Lam FM, Lau RW, Chung RC, Pang MY. The effect of whole body vibration on balance, mobility and falls in older adults: a systematic review and meta-analysis. Maturitas. 2012;72:206-13.

35. Sitjà-Rabert M, Rigau D, Fort Vanmeerghaeghe $A$, Romero-Rodríguez $D$, Bonastre Subirana M, Bonfill X. Efficacy of whole body vibration exercise in older people: a systematic review. Disabil Rehabil. 2012;34(11):88393. http://dx.doi.org/10.3109/09638288.2011.626486

36. Machado A, García-López D, González-Gallego J, Garatachea N. Whole-body vibration training increases muscle strength and mass in 
older women: a randomized-controlled trial. Scand J Med Sci Sports. 2010;20(2):200-7. http://dx.doi.org/10.1111/j.1600-0838.2009.00919.x

37. Van Nes IJ, Latour H, Schils F, Meijer R, Van Kuijk A, Geurts AC. Longterm effects of 6-week whole-body vibration on balance recovery and activities of daily living in the postacute phase of stroke. A randomized, controlled trial. Stroke. 2006;37:2331-5. http://dx.doi.org/10.1161/01.STR.0000236494.62957.f3

Referencias totales citadas: 37

Referencias citadas correspondientes a la Rev lb CC Act Fis Dep: 0 